\title{
Elaboración y Clasificación Sensorial de Gelatinas de Patas de Pollos. Correlación usando Redes Neuronales Artificiales
}

\author{
Poliana F. Almeida ${ }^{(1,2)}$, Wonder A. L. Alves ${ }^{(1)}$, Thiago M. B. Farias ${ }^{(1)}$, José C. Curvelo \\ Santana $a^{\left(1^{*}\right)}$
}

(1) Programa de Mestrado em Engenharia de Produção, Universidade Nove de Julho (UNINOVE),

Av. Francisco Matarazzo, 612, CEP: 05001-100, São Paulo-Brasil. (e-mail: jccurvelo@uninove.br)

(2) Instituto Federal de Educación, Ciencia y Tecnología Mato Grosso,IFMT - Campus São

Vicente, MT-Brasil. (e-mail: poliana.fernandes@hotmail.com)

Recibido Abr. 27, 2012; Aceptado Jun. 19, 2012; Versión final recibida Jul. 06, 2012

\section{Resumen}

Se presenta la estrategia para desarrollada para la clasificación sensorial de gelatinas obtenidas de patas de pollos utilizando redes neuronales basadas en los algoritmos de Kohonen. Estas redes muestran ser buenas herramientas para hacer las comparaciones sensoriales entre muestras, permitiendo identificar cuáles son las mejores muestras entre las gelatinas. De acuerdo a los resultados la Gelatina D, con $3,80 \%(\mathrm{~m} / \mathrm{v})$ de polvo de gelatina y $28,6(\mathrm{~m} / \mathrm{v})$ de azúcar fue a mejor entre las gelatinas de patas de pollo, recibiendo una aceptación sensorial entre 6 y 8 puntos en la escala hedónica.

Palabras clave: redes neuronales, gelatinas de patas de pollos, análisis sensorial, calidad de los alimentos

\section{Preparation and Sensorial Classification of Gelatins from Chicken Feet. Correlation using Artificial Neural Networks}

\begin{abstract}
A strategy has been developed for the sensorial classification of jellies made from chicken feet using neural networks based on Kohonen algorithms. These networks showed to be good tools for sensorial comparison among samples, allowing identification of the best gelatins. The sample Gelatin D, con $3.80 \%(\mathrm{w} / \mathrm{v})$ of gelatin powder and $28.6(\mathrm{w} / \mathrm{v})$ of sugar was the best than gelatin samples from chicken feet, with its sensorial qualities varying between 6 and 8 times in hedonic scale.
\end{abstract}




\section{INTRODUCCIÓN}

Las redes neuronales artificiales (RNA) han contribuido a los diversos campos de la investigación en la solución de problemas complejos. En la ingeniería mecánica, cómo se ha señalado en trabajo desarrollado por Villada y Cadavid (2007), que han presentado un algoritmo para diagnosticar fallas entre espiras del estator de motores de inducción mediante la aplicación de RNA y por Alves et al. (2011) han presentado una metodología para análisis de la rugosidad basada en las características superficiales de las imágenes obtenidas de microscopios ópticos y electrónicos, donde los valores extraídos de los patrones de rugosidad primaria se incorporan después a una RNA de perceptrón multicapa. En la ingeniería de organización, cómo el trabajo levado a cabo por Henriquéz y Palma (2011) se detalla el mecanismo automatizado por RNA por el cual se determina el estado de la iluminación, temperatura, humedad y otros valores de entorno y cómo se actúa sobre los patrones de comportamiento del usuario. En las ciencias naturales y da tierra, cómo el trabajo de García et al. (2010), que han aplicado un modelo de RNA con retropropagacion, para predecir el transporte de contaminantes (cobre y cadmio) en medios saturados, homogéneos e isotrópicos para diferentes clases texturales. En la ciencia de alimentos, como el trabajo de Curvelo Santana et al. (2010), que han presentado una RNA basada en los algoritmos de Kohonen para lo agrupamiento de vinos de cerezas de los Barbados por sus calidades sensoriales y en la medicina, como el trabajo desarrollado por Dias y Radonsky (2003), que han presentado una RNA basada en los algoritmos de Kohonen para la identificación de las enfermedades por el agrupamiento de sus síntomas. Todos estos autores han demostrado que las RNA son buenas herramientas para aplicación en el control, en la inspección de la calidad de productos, en los diagnósticos medicinales, en las organizaciones de empresas y en otros campos de la investigación, donde se obtiene buenos resultados.

En la actualidad, existe una preocupación creciente acerca de la preservación del medio ambiente. Así, varias investigaciones para ver cómo reducir los impactos ambientales causados por la eliminación de residuos industriales favorece la preservación de los componentes del agua y del suelo en todo el mundo (Coria y Vegas, 2011; Passarini et al., 2012; Rodríguez et al., 2008; Rosa et al., 2010). Los mataderos generan efluentes líquidos compuestos de grasas, proteínas y restos de animales. También generan los desechos sólidos, tales como: los huesos, las entrañas, patas, cabezas, piel y otros restos de carcasas. En este contexto, se hace necesario buscar alternativas de aprovechamiento y reutilización de los residuos obtenidos en los mataderos. En Brasil, la industria considera a las extremidades del pollo (patas) como un subproducto de escaso interés comercial. Los precios por este subproducto son muy bajos, debido a que la población no tiene interés en consumirlo. También, gran parte de este producto es desechado como un residuo, por falta de un mercado y otra parte es utilizado en la fabricación de los alimentos para animales (Almeida et al., 2012a y 2012b). De acuerdo con Gómez-Guillén et al. (2011) y Karim y Bhat (2009) una alternativa a la valorización de residuos es el desarrollo de nuevos productos dando a los residuos un destino más noble y de mayor valor comercial.

La gran mayoría de los colágenos comerciales son procedentes de mamíferos y son obtenidos principalmente de la piel del cerdo y del ganado. Mas, por razones socio-culturales e religiosos, productos de la piel del cerdo no son aceptables para el judaísmo y el Islam y los productos de carne de res son aceptables sólo si se ha tramitado de acuerdo con los requisitos religiosos (Giménes et al., 2009; Gómes-Gulién et al., 2011; Karim and Bhat, 2009). Adicionalmente, ocurre que con frecuencia la encefalopatía espongiforme bovina (EEB) y diversas enfermedades en la boca. Estos han sido serios problemas para la salud humana y por lo tanto los subproductos de los mamíferos están limitados a la utilidad de procesamiento de alimentos funcionales, productos cosméticos y farmacéuticos (Gómes-Gulién et al., 2011; Karim y Bhat, 2009). Es importante destacar que, durante el proceso de raspado de piel de vacuno utilizado en la producción de colágeno, se producen residuos contaminante que contienen cromo (Gómez-Guillén et al., 2011; Serna-Cock et al., 2010). Ante esta problemática, diversas investigaciones muestran otras alternativas de extracción de colágeno a partir de fuentes marinas, como por ejemplo: del esturión (Acipenser baeri) (Hao et al., 2009); de la raya (Raja kenojei) (Gómez-Guillén et al., 2011); del calamar gigante (Dosidicus gigas) (Giménez et al., 2009); de salmón del Atlántico (Salmo salar) (Arnesen y Gildberg, 2007); de la corvina sin (Johnius dussumieri); del marrajo jurel (Decapterus 
macrosoma) (Cheow et al., 2007); del bagre de canal (Ictalurus punctatus) (Yan et al., 2007) y otros peces (Gómez-Guillén et al., 2011; Karim y Bhat, 2009). Así, este trabajo se presenta la estrategia para desarrollada para la clasificación de las cualidades sensoriales de gelatinas obtenidas de las patas de pollos utilizando las redes neuronales de Kohonen.

\section{MATERIALES Y MÉTODOS}

Como materia prima para la elaboración de gelatina se utilizaron patas de pollos recién sacrificados en el Instituto Federal de Educación, Ciencia y Tecnología Mato Grosso - IFMT Campus São Vicente. Fueron lavadas las patas, después fueron retiradas las uñas y luego se lavaron de nuevo las patas con agua fría para eliminar cualquier residuo de suciedad. Entonces, las patas limpias fueron sometidas al proceso de cocción a $60^{\circ} \mathrm{C}$ durante $8 \mathrm{~h}$. Después de la cocción, la porción líquida resultante fue separada de las grasas en un filtró con algodón y fue secada en estufa con circulación de aire. La muestra secada se colocó en un recipiente de vidrio y se molió para obtener un polvo de gelatina. Se añadieron $100 \mathrm{~mL}$ de agua y se produjo las muestras de gelatinas formulados con sabor a uva, las composiciones están presentadas in la Tabla 1. A las muestras de gelatinas se añadieron $0,5 \mathrm{~g}$ de conservante sorbato de potasio y $10 \mathrm{~g}$ de polvo Siber® sabor a uva. Este tiene en su composición colorantes, almidón, acidulante, regulador de la acidez y anti-humectante (Almeida et al., 2012 b). La gelatina comercial utilizada fue de la marca Dr. Oetker, con sabor a uva $(\mathrm{H})$, producida de acuerdo con su fabricante (Arnesen y Gildberg, 2007; Almeida et al., 2012a y 2012b; Karim y Bhat, 2009; Liu et al., 2001; Yan et al., 2007). Las análisis de la composición de la gelatina fueron hachas de acuerdo con Arnesen y Gildberg (2007); Cheow et al. (2007); Hao et al. (2009) y Karim y Bhat (2009).

Tabla 1: Composición de las gelatinas de patas de pollo experimentales.

\begin{tabular}{lcc}
\hline Muestras & Polvo de gelatina $(\mathrm{g} / 100 \mathrm{~mL})$ & Azúcar $(\mathrm{g} / 100 \mathrm{~mL})$ \\
\hline Gelatina A & 1,903 & 7,1 \\
Gelatina B & 3,806 & 7,1 \\
Gelatina C & 1,903 & 28,6 \\
Gelatina D & 3,806 & 28,6 \\
\hline
\end{tabular}

La evaluación sensorial fue realizada por cincuenta catadores no entrenados. Todos fueron estudiantes del campus del IFMT, Campus São Vicente. El equipo incluyó a individuos de ambos sexos, con edades comprendidas entre 15 y 25 años. Las muestras fueron servidas de forma monódica, en cabinas individuales en vasos de plástico desechables codificados con cantidades estandarizadas. Los catadores tenían a su disposición un vaso de agua que se utiliza para enjuagar la boca entre las muestras y las respuestas fueron registradas en una tabla de respuestas estandarizada. Las pruebas afectivas se utilizan cuando se necesita conocer el "estado afectivo" de los consumidores por el producto, y para ello se utilizan escalas hedónicas. De los valores relativos de aceptación se puede inferir la preferencia. Es decir, las muestras más aceptadas son las más preferidas. Así, se evaluaron los atributos de apariencia, sabor, aroma, textura e incluso la disponibilidad de consumir el producto. Se utilizó la prueba con la escala afectiva hedónica de 9 puntos, que van desde 1-no les gusta muchísimo a 9-les gustó muchísimo. Con los resultados obtenidos de los cuestionarios se tabularon las respuestas en orden de frecuencia. El método de $t$ Student fue utilizado para el análisis estadísticas de las muestras (Alvis et al., 2008 y 2011; Costa et al., 2008; Curvelo Santana et al., 2010; Ronceros et al., 2008; Rodrígues y Gualdron, 2011).

Teniendo en cuenta estas consideraciones, se presenta los resultados de las analices sensoriales por el conjunto $\zeta=\left\{\left(c_{i}, p_{i}\right) \in \mathrm{Z}^{4} \times\{A, B, \ldots, H\}: i=1,2, \ldots, 50\right\}$ de manera que un elemento $X_{i}=\left(c_{i}, p_{i}\right) \in \zeta$ as una secuencia de evaluaciones $c_{i}$ (de aroma, sabor, textura e apariencia) del producto $p_{i}$ por el catador $i$. Por lo tanto, sus similitudes son presentadas en el resultado de la 
analice sensorial $\zeta$ por el mapas de Kohonen. Inicialmente, se consideró que los resultados de las evaluaciones individuales de cada calidad sensorial (aroma, sabor, textura y apariencia), en otras palabras, elles fueron presentados por el conjunto $\zeta_{j}=\left\{\left(c_{i}^{j}, p_{i}\right) \in \zeta: j\right.$ é um índice do vetor $\left.c_{i}\right\}$ y después se hizo un análisis exhaustivo de todos resultados sensoriales. Para la analices de los conjuntos $\zeta_{\text {y }} \zeta_{j}$ (con $\left.1 \leq j \leq 4\right)$ se utilizaron las redes neuronales de Kohonen, que se hizo una búsqueda en la tabla de datos sensoriales, que fueron utilizados como las entradas $\left(\zeta \circ \zeta_{j}\right)$ en la estructura $Y$, de tal manera que cuando se presenta una entrada $X_{i}=\left(X_{1}, \ldots X_{n}\right) \in \zeta$ para la rede neuronales, un neurona $y_{1} \in Y$ fue elegido para representar la rede mediante a un proceso de competición y, así, la similitud de lo neurona elegido $y_{1}$ y su vecino topológico fue aumentada y por lo tanto, se obtienen los mapa de Kohonen tal como se muestra en la Fig. 1 (Curvelo Santana et al., 2010; Dias y Radonsky, 2003; Kohonen, 1989). En esta construcción de la RNA, también conocido como el proceso de formación, los parámetros se consideran: la arquitectura de la RNA (tamaño del vector de entrada y la estructura topológica), tasa de aprendizaje y el número de iteraciones del algoritmo de entrenamiento. En este caso, se utilizó $1 \times 18$ y 5x5, con tasa de aprendizaje de 0,0005 y 500 iteraciones. Después de la construcción de la RNA se analizaron las estructuras topológicas de las neuronas de cada RNA para determinar las similitudes entre los conjuntos de datos $\zeta$ o $\zeta_{j}$.

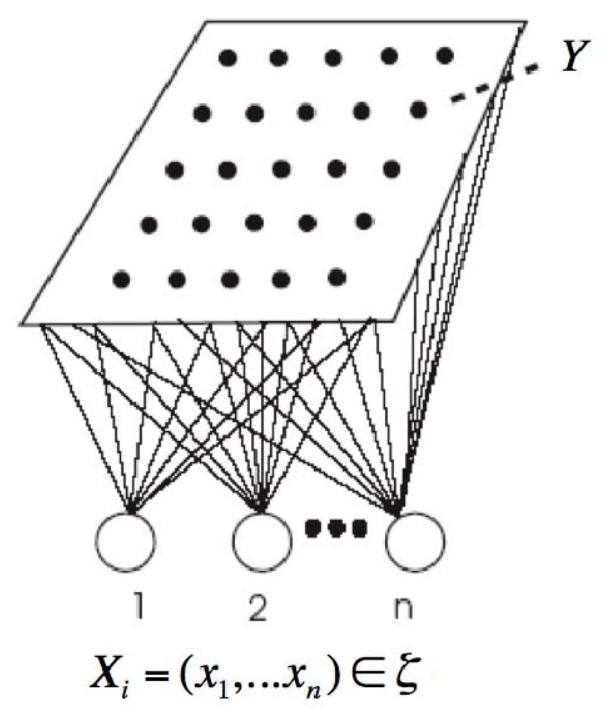

Fig 1: Mapa de Kohonen.

\section{RESULTADOS Y DISCUSIÓN}

La Tabla 2 muestra la composición de las patas y gelatinas de pollos. Después de la extracción y secado, fue obtenido un rendimiento de $5,337 \%$ de masa de gelatina, con relación a la masa de las patas de pollo. Que es de 78,5\% de proteínas, 9,7\% de humedad, 6,9 de grasas y 4,85\% de cenizas. Hao et al. (2009) han obtenido un rendimiento entre 2,40-3,52 \% para la gelatina de píele de estrujón y Jamilah \& Hervinder (2002) han obtenido un rendimiento entre 5,39 \% para la gelatina de píele de tilapia negra. Entretanto, Cho et al. (2009) han obtenido un rendimiento entre $17,48 \%$ para la gelatina de píele de rayo (Raja kenojei) y Gimenez et al. (2009) han obtenido un rendimiento entre $12 \%$ para la gelatina de píele de calamar gigante. Almeida et al. (2012 b) muestro que demuestra una calidad superior de la gelatina obtenida de las patas de pollo con respecto a la gelatina comercial. Por lo tanto, el consumo diario de una gelatina de las patas de pollo es equivalente al consumo de cuatro gelatinas comerciales, manteniendo las mismas propiedades nutricionales y medicinales. Así, los productos obtenidos tienen una buena calidad sensorial y química. También la materia prima tiene un precio de bajo y es más aceptable para los aspectos de la salud del ser humano que la obtenida de la piel de cerdo y ganado vacuno. 
Finalmente tiene una mayor aceptación por las comunidades judaica y el Islam (Almeida et al., 2012.b; Gómes-Gulién et al., 2011; Karim y Bhat, 2009).

Tabla 2: Composición centesimal de las muestras de patas y gelatinas pollos

\begin{tabular}{lcccc}
\hline \multirow{2}{*}{ Parámetro Analizado } & \multicolumn{2}{c}{ Patas de Pollos } & \multicolumn{2}{c}{ Gelatina } \\
\cline { 2 - 5 } & $\mathrm{g} / 100 \mathrm{~g}$ & \pm & $\mathrm{g} / 100 \mathrm{~g}$ & \pm \\
\hline Humedad & 60,009 & 3,182 & 9,749 & 0,846 \\
\hline Cenizas & 9,943 & 1,694 & 4,807 & 1,839 \\
\hline Lípidos & 12,875 & 1,928 & 6,919 & 1,401 \\
\hline Proteína & 17,173 & 1,781 & 78,525 & 1,650 \\
\hline Rendimiento de las extracción & & & 5,337 & 0,051 \\
\hline
\end{tabular}

La Tabla 3 muestra los valores medios del análisis sensorial de las gelatinas de patas de pollo y de la muestra comercial. Los promedios obtenidos por la gelatina comercial fueron superiores a 7 para todas las calidades sensoriales, lo que indica que la mayoría de los catadores dijeron haber gustado de la gelatina. En cuanto a mayoría las gelatinas de patas de pollo los valores fueron menores a 4; siendo que apenas la muestra "Gelatina D" han presentado calidades sensoriales a la cerca de 6, lo que indica que la mayoría de los catadores, dijo que "gustaba poco" de esta gelatina. El análisis estadístico utilizando lo teste de $t$ Student mostró que no hubo diferencias significativas entre las muestras para todas las cualidades, aunque algunas muestras recibieron promedios ton bajas. Este efecto se produce porque muchos análisis (cincuenta) se utilizaron para cada muestra, diluyendo el efecto en sus desviaciones estándar.

Tabla 3: Evaluación de las cualidades sensoriales de las gelatinas de las patas de pollo.

\begin{tabular}{lccccc}
\hline \multirow{2}{*}{ Muestras } & \multicolumn{5}{c}{ Promedio de los Valores Sensoriales } \\
\cline { 2 - 6 } & Apariencia & Aroma & Sabor & Textura & Aspecto General \\
\hline Gelatina A & $3,76^{\mathrm{a}}$ & $2,68^{\mathrm{b}}$ & $2,60^{\mathrm{c}}$ & $2,32^{\mathrm{d}}$ & $2,8^{\mathrm{e}}$ \\
Gelatina B & $4,60^{\mathrm{a}}$ & $3,10^{\mathrm{b}}$ & $2,70^{\mathrm{c}}$ & $5,88^{\mathrm{d}}$ & $3,72^{\mathrm{e}}$ \\
Gelatina C & $4,38^{\mathrm{a}}$ & $4,16^{\mathrm{b}}$ & $4,18^{\mathrm{c}}$ & $3,64^{\mathrm{d}}$ & $3,64^{\mathrm{e}}$ \\
Gelatina D & $5,92^{\mathrm{a}}$ & $5,30^{\mathrm{b}}$ & $5,76^{\mathrm{c}}$ & $6,76^{\mathrm{d}}$ & $6,06^{\mathrm{e}}$ \\
Gelatina Comercial & $7,32^{\mathrm{a}}$ & $7,36^{\mathrm{b}}$ & $7,34^{\mathrm{c}}$ & $7,20^{\mathrm{d}}$ & $7,26^{\mathrm{e}}$ \\
\hline Obs: mismas letras significa que no hay diferencia entre las muestras a un nivel de confianza de $95 \%, t_{0,05,(98)}=1,66$
\end{tabular}

En la Fig 2 se presenta los mapas de Kohonen obtenidos de los datos experimentales de las calidades sensoriales das gelatinas. Mirando los mapas resultantes de la aplicación de las redes de Kohonen sobre los conjuntos de datos $\zeta_{j}$ se observo una formación de los tres grupos predominantes, a 8 , a 6 y a 2 puntos en la escala hedónica. La muestra comercial es similar a la muestra Gelatina D en las cualidades apariencia y textura. La muestra Gelatina D es similar a la muestra Gelatina C en las calidades aroma y sabor. Las muestras Gelatina A y B son mucho similares en la mayoría de sus calidades sensoriales. Dóndes se puede señalar que la Gelatina comercial fue buena evaluada con promedios a cerca de 8 para todas las calidades sensoriales, la muestra Gelatina C tuvo su puntuación entre 2 y 6 , mientras las Gelatinas A y B tuvo las puntuaciones más bajas, un promedio a cerca de 2. Las Gelatina D fue la más buenas evaluadas entre las gelatina de patas de pollo, con un promedio entre 6 y 8 en escala hedónica para todas las calidades sensoriales. Así, se nota que los mapas de Kohonen fueron más eficaces que la técnica estadística, conseguir una mejor clasificación de las muestras y permitiendo hacer una mejor comparación y identificar cuáles son las mejores muestras. Esto hace que sea posible identificar cuál es la mejor composición de gelatina para uso en el mercado. Este comportamiento también fue verificado por Curvelo Santana et al. (2010) para las calidades de vinos de cerezas del Barbados. 


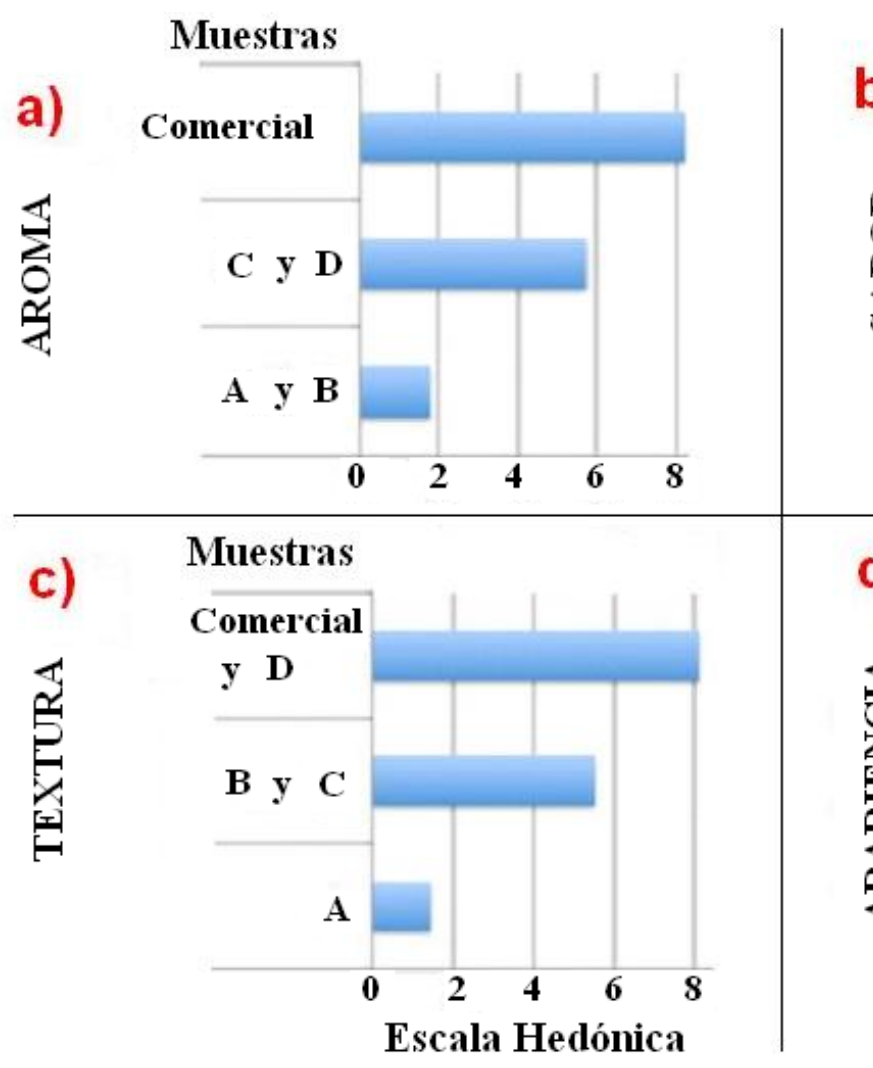

Grupos
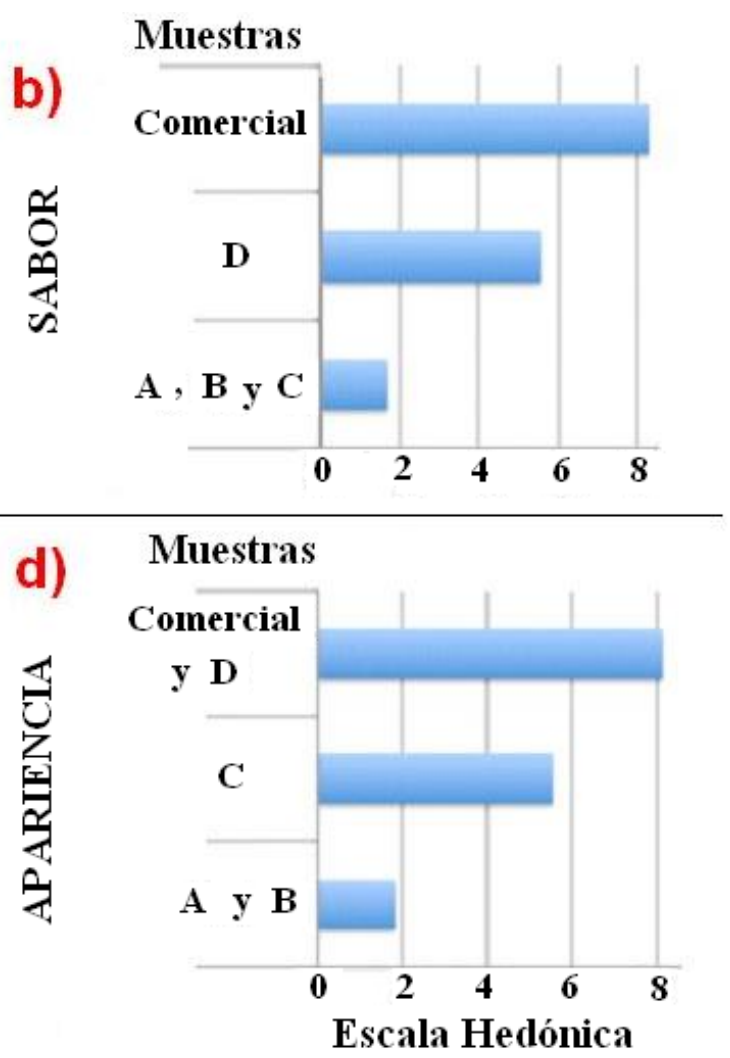

Puntos en la escala hedónica

Fig. 2: Los resultados de los agrupamientos obtenidos de las redes neuronales de Kohonen. Para las calidades: a) aroma, b) sabor, c) textura d) apariencia.

De conformidad con Almeida et al. (2012.b) los niveles de proteínas en las gelatinas de patas de pollo superaron en cuatro veces los niveles de los productos comerciales, lo que indica una superioridad y con respecto a la calidad química. Como el consumo de gelatina es indicado por hacer el bien para la salud humana, la dosis diaria de gelatina podría reducirse en cuatro veces al utilizar la gelatina de las patas de pollo, sin perder sus propiedades medicinales. Por su consumo non está asociado a problemas socio-culturales, non transmitir enfermedad y por sus las cualidades químicas y sensoriales permiten la sustitución de las materias primas utilizadas actualmente por las patas de pollo (Giménes et al., 2009; Gómes-Gulién et al., 2011; Karim and Bhat, 2009). También se contribuir con el desarrollo sostenible, por la eliminación de los residuos generados en la matanza de pollos y explorando el potencial de la industria del tarso de pollo en la obtención de un alimento de alta calidad, así como con buenas prácticas de producción.

\section{CONCLUSIONES}

Las redes neuronales artificiales de Kohonen son buenas herramientas para hacer las comparaciones sensoriales entre muestras de gelatinas, permitiendo identificar cuáles son las mejores muestras entre ellos. De conformidad con el resultado global, la Gelatina D, con 3,80 \% $(\mathrm{m} / \mathrm{v})$ de polvo de gelatina y $28,6(\mathrm{~m} / \mathrm{v})$ de azúcar fue a mejor entre las gelatinas de patas de pollo, recibiendo una aceptación sensorial entre gustado moderadamente y gustado, que son correspondientes a 6 y 8 puntos en la escala hedónica.

\section{AGRADECIMIENTOS}

La UNINOVE y el Programa PROSUP/CAPES por el apoyo económico. 


\section{REFERENCIAS}

Almeida, P. F., M. G. O. Araújo y J. C. C. Santana, Collagen extraction from chicken feet for jelly production, Acta Scientiarum. Technology, 34(3), 345-351 (2012a).

Almeida, P. F. y otros 3 autores. Aprovechamiento de patas de pollos como alternativa para evitar los residuos generados en los mataderos, Información Tecnológica: 23(4), en prensa 2012b.

Alves, M. L, B. B. Ferreira, B. y F. R. Leta, Evaluación de parámetros de rugosidad usando análisis de imágenes de diferentes microscopios ópticos y electrónicos, Información Tecnológica: 22(4), 129-146 (2011).

Alvis, A., H. S. Villada y D. C. Villada, Efecto de la temperatura y tiempo de fritura sobre las características sensoriales del ñame (Dioscorea alata), Información Tecnológica: 19(5), 19-26 (2008).

Arnesen, J. A. y A. Gildberg, Extraction and characterisation of gelatin from Atlantic salmon (Salmo salar) skin, Bioresource Technology: 98, 53-57 (2007).

Cheow, C. S. y otros 3 autores, Preparation and characterisation of gelatins from the skins of sin croaker (Johnius dussumieri) and shortfin scad (Decapterus macrosoma), Food Chemistry: 101, 386-391 (2007).

Coria, I.D. y Vegas, M., Determinación de la Efectividad de la remediación de suelos accidentalmente contaminados con ácido sulfúrico mediante métodos geofísicos, Información Tecnológica: 22(1), 3-8 (2011).

Curvelo Santana, J. C. y otros 3 autores, Applying of neural network on the wine sensorial analysis from Barbados cherry, Journal of Food Process Engineering: 33(1), 365-378 (2010).

Dias, C. G. y V. Radonsky, The development of an auxiliary system to help in the pediatrics diagnosis using artificial neural. Exacta: 1(1), 89-95 (2003).

Giménez, B., y otros 4 autores, Physico-chemical and film forming properties of giant squid (Dosidicus gigas) gelatin, Food Hydrocolloids: 23, 585-592 (2009).

Gómez-Guillén, M. C. y otros 3 autores, Functional and bioactive properties of collagen and gelatin from alternative sources: A review, Food Hydrocolloids: 25, 1813-1827 (2011).

Hao, S., y otros 6 autores, The characteristics of gelatin extracted from sturgeon (Acipenser baeri) skin using various pretreatments, Food Chemistry: 115, 124-128 (2009).

Henríquez, M. R y P. A. Palma, Control automático de condiciones ambientales en domótica usando redes neuronales artificiales, Información Tecnológica: 22(3) 125-139 (2011).

Jamilah, B. y K. G. Harvinder, Properties of gelatins from skins of fish-black tilapia (Oreochromis mossambicus) and red tilapia (Oreochromis nilotica), Food Chemistry: 77, 81-84 (2002).

Karim, A. A. y Bhat, R. Fish gelatin: properties, challenges, and prospects as an alternative to mammalian gelatins. Food Hydrocolloids 23, 563-576 (2009).

Kohonen, T. Self-Organization and Associative Memory, Berlin: Springer-Verlag, (1989).

Liu, D. C., Y. K. Lin y M. T. Chen, Optimum condition of extracting collagen from chicken feet and its characteristics, Asian-Australasian Journal of Animal Science: 14, 1638-1644 (2001). 
Mendes, B. y otros 3 autores, Detección de posición angular de embarcaciones, utilizando técnicas de visión computacional y redes neurales artificiales. Información Tecnológica: 21(6) 177188 (2011).

Passarini, K. C., y otros 3 autores, Reutilización de las Aguas Residuales en la Irrigación de Plantas y en la Recuperación de los Suelos. Información Tecnológica: 23(1) 57-64 (2012).

Rivera, J. A., J. G. Sebranek y R. E. Rust, Functional properties of meat by-products and mechanically separated chicken (MSC) in a high-moisture model petfood system, Meat Science: 55, 61-66 (2000).

Rodríguez, J. F. R. y L. Gualdron, Evaluación de la sustitución de grasa animal por grasa vegetal Insaturada en la Elaboración de un Embutido de Carne de Búfalo (Bubalus bubalis), Información Tecnológica: 22, 43-54 (2011).

Ronceros, B.A. y otros 3 autores, Efecto de la temperatura y tiempo de almacenamiento sobre la calidad del tomate deshidratado, Información Tecnológica: 19(5), (2008).

Rosa, M. A., J. M. Peralta y D. M. Bosco, Estimación de parámetros cinéticos de la degradación aeróbica de efluentes lácteos usando AQUASIM v 2.1b, Información Tecnológica: 21(3) 51-56 (2010).

Santos, C.T., y otros 5 autores, Characterization and sensorial evaluation of cereal bars with jackfruit, Acta Scientiarum Technology: 33(1), 81-85 (2011).

Saxena, A. y otros 3 autores, Membrane-based techniques for the separation and purification of proteins: An overview, Adv Colloid Interfac.: 145(1-2), 1-22 (2009).

Serna-Cock, L., M. Velásquez y A. A. Ayala, Efecto de la ultrafiltración sobre las propiedades reológicas de gelatina comestible de origen bovino, Información Tecnológica: 21(3), 91-102 (2010).

Silva, F. A. S., M. E. M. Duarte y M. E. R. M. Cavalcanti-Mata, New methodology for data interpretation of food sensorial analysis, Engenharia Agrícola: 30(5), 967-973 (2010).

Villada, F. y D. R. Cadavid, Diagnostico de fallas en motores de inducción mediante la aplicación de redes neuronales artificiales, Información Tecnológica: 18(2), 105-112 (2007).

Yan, H. y otros 5 autores, 2-Step optimization of the extraction and subsequent physical properties of channel catfish (Ictalurus punctatus) skin gelatin. Journal of Food Science: 72(4), C188-C195 (2007). 